ENCYCLOPÉDIE Encyclopédie berbère

BERBERE

$31 \mid 2010$

31 | Matmora - Meẓrag

\title{
Métrique (de la poésie berbère traditionnelle)
}

\section{A. Bounfour}

\section{OpenEdition}

Journals

Édition électronique

URL : http://journals.openedition.org/encyclopedieberbere/584

DOI : 10.4000/encyclopedieberbere.584

ISSN : 2262-7197

\section{Éditeur}

Peeters Publishers

\section{Édition imprimée}

Date de publication : 30 décembre 2010

Pagination : 4941-4947

ISBN : 978-90-429-2368-3

ISSN : 1015-7344

\section{Référence électronique}

A. Bounfour, « Métrique (de la poésie berbère traditionnelle) », Encyclopédie berbère [En ligne], 31 | 2010, document M100, mis en ligne le 08 octobre 2020, consulté le 13 octobre 2020. URL : http://

journals.openedition.org/encyclopedieberbere/584 ; DOI : https://doi.org/10.4000/ encyclopedieberbere.584

Ce document a été généré automatiquement le 13 octobre 2020.

(c) Tous droits réservés 


\title{
Métrique (de la poésie berbère traditionnelle)
}

\author{
A. Bounfour
}

1 L'histoire des études sur la métrique de la poésie berbère peut être faite en quelques paragraphes. Plutôt qu'un exposé chronologique, on privilégiera les contenus de ces études en les classant selon qu'elles tiennent compte de tel ou tel élément linguistique comme base de la métrique ou des métriques de la poésie berbère. Les noms des auteurs baliseront la trame chronologique de ces études.

2 La littérature spécialisée est très restreinte. Jusqu'en 1984, date de la soutenance de la thèse d'Etat de A. Bounfour sur la métrique chleuhe, on recense six auteurs qui ont apporté une contribution significative dans le domaine de la métrique berbère. Les voici dans l'ordre chronologique : H. Stumme, Ch. de Foucauld, S. Boulifa, A. Basset, H. Jouad, M. Mammeri. La nature de ces études varie: un article plus ou moins long (Basset, Jouad et Mammeri) ou un chapitre dans un livre (Stumme, Foucauld, Boulifa).

\section{Les différentes conceptions de la métrique berbère}

3 Si l'on excepte le cas de H. Jouad, ces auteurs peuvent être classés en trois catégories selon l'élément fondamental à la base de leur métrique. En effet, Stumme, suivi par A. Basset, soutiennent la thèse selon laquelle la métrique berbère - chleuhe pour le premier et kabyle pour le second - repose sur l'accent, particulièrement l'accent de mot. S. Boulifa soutient que la métrique kabyle repose sur le nombre de syllabe et la rime. Quant à $\mathrm{Ch}$. de Foucauld, il montre que la métrique de la poésie touarègue repose sur la longueur syllabique.

Dans notre thèse (1984), nous avons évalué le pouvoir explicatif de chacune de ces métriques :

5 1. Deux symptômes fragilisent la métrique accentuelle de Stumme et de Basset: les analyses empiriques amènent le premier à postuler qu'une consonne seule peut jouer le rôle d'une syllabe et l'appelle d'ailleurs 'consonne-syllabe'; chez Basset, le symptôme 
est plutôt une contradiction : après avoir énoncé que l'accent ne peut tomber sur un clitique, il se voit obligé, dans l'analyse empirique, de faire le contraire. Dans les deux cas, les solutions proposées apparaissent comme des règles ad hoc et rendent la thèse inconsistante. A cela il faut ajouter que les études sur l'accent en berbère étaient inexistantes à l'époque et sont rares encore aujourd'hui.

6 2. La thèse de $\mathrm{S}$. Boulifa est plus consistante pour la poésie kabyle. En effet, le nombre de syllabes dans un asefru (poème de 9 vers distribués en 3 tercets), par exemple, est le suivant: $(7+5+7) \times 3$. L'analyse empirique confirme cette consistance sur un plan statistique : elle fonctionne dans un très grand nombre de cas. On ne retiendra pas la rime comme constitutive de la métrique puisqu'on ne la trouve nulle part ailleurs excepté en rifain. La rime est un simple marquage de la fin du vers; elle est plus de l'ordre de la ponctuation que de la consistance métrique. On peut d'ailleurs soutenir la thèse selon laquelle il s'agirait d'un emprunt à la poésie arabe dialectale et affirmer que le distique chleuh ou le tercet kabyle sont, originairement, un vers composé de deux hémistiches pour l'un et de trois hémistiches pour l'autre (cf. Bounfour 1999, p. 229-231). En voici deux illustrations :

1. Chleuh:

a. A ur irhem rbbi abnnay mqqar igg ${ }^{\circ}$ ez akal

Acku nttan a nn-igan afrig i izenk ad

b. A ur irhem rbbi abnnay mqqar igg ${ }^{\circ}$ ez akal//acku nttan a nn-igan afrigi izenk ${ }^{\circ} a d$

c. Que Dieu ne puisse pardonner au maçon! Qu'il trépasse !

Car c'est lui qui a entouré de haie les mouflons.

2. Kabyle :

a. Șllu sala Muhemmed

Alef wella yazid

Zidu ș̣lat eal Lhadi

I wul-nner ad yettberred

Glebher ljamed

A $\gamma$ yeșleh menna w radi

b. Șllu sala Muhemmed / alef wella yazid / Zidu șslat Eal Lhadi

I wul-nney ad yettberred / g lebher ljamed / a y yeșleh menna w radi

c. Béni soit le Prophète / mille fois et plus / béni soit le Guide !

7 Les deux présentations $1 \mathrm{a}$ et $2 \mathrm{a}$ sont habituelles dans la tradition qui utilise la transcription latine alors que les présentations $1 b$ et $2 b$ sont canoniques dans les transcriptions en caractères arabes anciennes - les manuscrits chleuhs et kabyles en témoignent - et actuelles puisque les publications, massivement marocaines, le prouvent.

On notera que l'exemple chleuh a une fin de vers ou d'hémistiche sans rime alors que le poème kabyle est rimé. Il est même monorimique dans $2 b$, comme c'est le cas en arabe.

9 3. La thèse de Ch. de Foucauld nous était apparue plus solide que les deux précédentes à condition d'être réinterprétée selon les données linguistiques de la langue berbère dont le touareg. En effet, Ch. de Foucauld soutient l'idée que le vers consiste en une distribution de trois types de syllabe : une syllabe brève, une longue et une très longue qu'il appelle 'syllabe traînée'. L'interprétation que nous avions donnée de cette thèse était celle-ci : puisqu'il n'y a pas, globalement, d'opposition vocalique en berbère (voyelle brève/voyelle longue), y compris en touareg, malgré quelques rares cas dans un contexte morphologique particulier, la syllabe dite traînée n'apparaissant qu'en fin de vers est un élément de ponctuation; l'opposition syllabe brève/syllabe longue apparaît dans l'analyse empirique et de manière systématique comme une opposition 
de syllabe $\mathrm{x}$ à une syllabe fermée à voyelle pleine. Il ne reste plus qu'à identifier en quoi consiste cette syllabe $\mathrm{x}$ : elle est ou ouverte à voyelle pleine $(\mathrm{CV})$ ou fermée à voyelle ultra-brève (le schwa, cəc). Nous avions proposé d'appeler cette dernière "syllabe légère " et l'autre "syllabe lourde ». Quant à la syllabe dite trainée, elle est obligatoirement ou lourde ou surlourde (doublement fermée, CVC ou CVCC). Cette dernière ne peut occuper aucune autre place que la fin du vers.

Cette réinterprétation autorise à qualifier cette métrique de quantitative à condition d'exclure la quantité vocalique et de ne retenir que la quantité syllabique.

11 La thèse de Ch. de Foucauld, réinterprétée, permet de postuler une métrique unifiée pour de nombreux dialectes; ce qui permet d'entrevoir et d'expliquer la variation métrique selon les régions dialectales. On y reviendra.

On aura noté que les trois thèses précédentes sont formulées par des descripteurs de langue. La thèse de la syllabe dite traînée chez $\mathrm{Ch}$. de Foucauld est due à son écoute du chant et non exclusivement à sa connaissance de la phonétique touarègue. Or, H. Jouad, travaillant avec B. Lortat-Jacob, un ethnomusicologue, avait la possibilité d'explorer avec des moyens importants l'intuition de Ch. Foucauld. Le noyau central de sa thèse consiste à partir de la terminologie autochtone. Ce qui, en soi, est louable et pouvait être décisif. Le concept métrique autochtone fondamental est celui de tiyat en chleuh, que Jouad explicite comme étant des syllabes métriques. Certes, elles le sont d'une certaine manière: les modèles de tiyat sont en réalité des notations de syllabes 'marquées' ou non marquées pour le chant. Autrement dit, tyat ou 'frappes' est une écriture musicale qui dit que telle syllabe sera brièvement chantée, telle autre allongée, et telle autre très allongée. On voit que nous ne sommes pas très loin de $\mathrm{Ch}$. de Foucauld.

13 La non distinction de ce qui est métrique et de ce qui est rythme musical se présente aussi dans un domaine proche du berbère, la poésie maghrébine en arabe dialectal, voire même de la poésie arabe ancienne. Cette non différenciation des deux systèmes implique, chez Jouad, une certaine fragilité de sa métrique.

14 Pour terminer cette revue des thèses métriques, on signalera deux thèses qui viennent d'être soutenues: l'une sur la métrique kabyle (Salhi 2007) et l'autre sur la poésie tamazighte du Moyen Atlas marocain (Kich 2007). La première est une reformulation méthodique des thèses précédentes qui prend en compte l'analyse de la présentation typographique des poèmes. La seconde n'a d'autre intérêt que les 120 pages environ de corpus.

\section{Les règles métriques et les types de vers}

On peut postuler des règles métriques générales, c'est-à-dire applicables à toute poésie berbère traditionnelle ainsi que des règles spécifiques à chaque tradition ou à chaque région dialectale.

Les règles générales sont fondées sur un principe de base: la syllabe. La métrique berbère est syllabique. C'est pourquoi on peut dire que la thèse de Basset est syllaboaccentuelle - elle combine l'accent à la syllabe - et celle de Foucauld syllaboquantitative parce qu'elle combine la syllabe à sa quantité. La question première est donc celle-ci : comment scander syllabiquement un vers berbère? Autrement dit, comment reconnaitre les syllabes formatrices d'un vers? Il serait fastidieux dans le 
cadre limité d'une notice d'encyclopédie de décliner ces règles (cf. Bounfour 1999, p. 113-131).

17 La syllabation du vers permet de distinguer deux grandes traditions métriques: la tradition syllabo-quantitative et la tradition syllabo-rimique.

La première domine dans l'aire chleuhe, tamazighte et touarègue; la seconde est massive en kabyle et en rifain. Notons que toutes les deux combinent la syllabe au nombre : le vers chleuh, tamazight ou touareg obéit à la loi du nombre dans sa forme isométrique ; il en est de même en kabyle et en rifain même si dans la poésie kabyle on peut constater des variations notables. Enregistrons pour le moment que la différence entre les deux traditions se situe en fin de vers: la tradition quantitative utilise la syllabe lourde ou surlourde pour ponctuer le vers alors que la tradition syllabique utilise la rime. Illustrons cela par les exemples précédents :

\begin{tabular}{|c|c|}
\hline a. Șllu Eala Muhemmed & șl lu ea la mu ḥem med \\
\hline Alef wella yazid & a lef wel la ya zid \\
\hline Zidu șșlat Eal Lhadi & zi dușș lat ea lal ha di \\
\hline I wul-nney ad yettberred & i wu lenn yad yet ber red \\
\hline G lebher ljamed & eg leb herl ja med \\
\hline A $\gamma$ yeșleh menna $w$ radi & ar yeșleh men naw ya di \\
\hline
\end{tabular}

ou selon l'autre présentation :

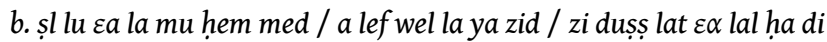
$i$ wu lenn yad yet ber red / eg leb herl ja med / a y yeș leh men naw ya di

2. Chleuh:

A ur irhem rbbi abnnay mqqar igg ${ }^{\circ}$ z akal / aw rir hem reb ba ben nay meq qa rig za kal Acku nttan a nn-igan afrig i izenkad / ac ku net ta na nni ga naf ri giy zen kad. ou

aw rir hem reb ba ben nay meq qa rig za kal / ac ku net ta na nni ga naf ri giy zen kad

Il y a une seconde différence située au niveau des types de vers. En effet, le vers est défini dans la tradition syllabo-rimique par le nombre de syllabes : on rencontre ainsi des vers de 5 et 7 syllabes en kabyle. En revanche, la tradition syllabo-quantitative divise le vers en plusieurs pieds; chaque pied est composé d'au moins deux syllabes dont la dernière est toujours une syllabe lourde. C'est ainsi que l'on rencontre des vers du type 2+4+6 syllabes, c'est-à-dire que ce vers est composé de trois pieds dont le premier est de deux syllabes, le second de 4 syllabes et le dernier de 6 syllabes. Cette tradition maintient le nombre de syllabes (l'isométrie souvent) comme la précédente : c'est ainsi que le vers hypothétique précédent $(2+4+6$ syllabes) est un 12 -syllabes auxquelles est articulé un niveau entre la syllabe et le vers, celui du pied.

24 3. Kabyle : 


\begin{tabular}{|c|c|}
\hline a. Șllu cala Muhemmed & șl lu ea la mu hem med \\
\hline Alef wella yazid & a lef wel la ya zid \\
\hline 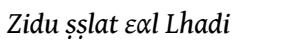 & zi dușs lat ca lal ha di \\
\hline I wul-nney adyettberred & $i$ wu lenn $\gamma$ ad $y$ et ber red \\
\hline G lebher ljamed & eg leb herl ja med \\
\hline A $\gamma$ yeșleḥ menna $w$ radi & ar yeș leḥ men naw ya di \\
\hline
\end{tabular}

ou

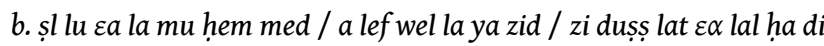

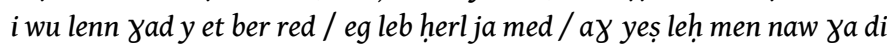

4. Chleuh :

a. A ur irhem rbbi abnnay mqqar igg ${ }^{\circ}$ z akal / aw rir hem reb ba ben nay meq qa rig za kal Acku nttan a nn-igan afrig i izenkad ac ku net ta na nni ga naf ri giy zen kad ou

b. aw rir hem reb ba ben nay meq qa rig za kal / ac ku net ta na nni ga na fri giy zen kad

Dans 3a, le premier et le dernier vers de chaque tercet sont les 7-syllabes et celui du milieu un 5 -syllabes; dans 3b, il s'agit de deux vers isométriques de 19 syllabes chacun regroupés en 3 hémistiches de 7, 5 et 7 syllabes ou 7+5+7 syllabes.

En $4 \mathrm{a}$, les deux vers sont des 12-syllabes dont la structure en pieds est la suivante :

i. en début de vers une syllabe lourde est convertie en 2 syllabes légères ; par conséquent, aw et ac compte chacune pour deux syllabes ;

ii. chaque vers est alors scandé en $8+3+2$ syllabes. On constate ainsi un double parallélisme, le premier est fondé sur l'isométrie et le second sur la place toujours la même de la syllabe lourde.

Telles sont les règles les plus fondamentales de la métrique berbère. Pour les détails, on se reportera aux études citées en bibliographie.

\section{BIBLIOGRAPHIE}

Nous n'avons retenu que les travaux significatifs dans l'élaboration d'une métrique générale ou particulière à tel ou tel dialecte berbère. Les applications de telle ou telle métrique, par les musicologues ou ethnomusicologues par exemple, ne sont pas citées.

BASSET A., 'Sur la métrique berbère', Comptes rendus sommaires de l'Institut français d'Anthropologie, fasc 6/80-86, 1952, p. 4-5. Repris dans : Etudes et documents berbères, 2, 1987, p. 83-90. 
BASSET A., « Remarques sur la métrique berbère dans quelques vers kabyles », Repris dans : Etudes et documents berbères, 5, 1989, p. 5-21.

BOULIFA A. S., Recueil de poèmes kabyles, Awal, Alger, 1990.

BOUNFour A., Le nœud de la langue. Langue, littérature et société au Maghreb, Edisud, 1994, p. 123-131. BOUNFOUR A., Linguistique et littérature : études sur la littérature orale marocaine, Thèse de doctorat d'Etat, Université de la Sorbonne Nouvelle-Paris III, 1984.

BOUNFOUR A., Introduction à la littérature berbère. 1. La poésie, Editions Peeters, 1999, p. 113-211.

DELL F. et ELMEDLAOUI M., Poetic Meter and Musical Form in Tashlhit Berber Songs, Berber Studies, volume 19, Rüdiger Koppe Verlag, Köln, 2006.

FOUCAULD Ch. (de), Poésies touarègues (dialecte de l'Ahaggar), Paris, 1925 et 1930.

JOUAD H., Le calcul inconscient de l'improvisation. Poésie berbère - Rythme, nombre et sens, Editions Peeters, 1995 ( $c f$. le compte-rendu de A. Bounfour dans Langues et littératures berbères. Chroniques des études XIII (1994-1995), L'Harmattan/INALCO, 1996, p. 36-47.

JOUAD H., 'Les éléments de la versification en berbère marocain', Thèse de doctorat de $3^{\mathrm{e}}$ cycle, Université de la Sorbonne Nouvelle-Paris III, 1983.

JOUAD H., 'Mètres et rythmes de la poésie orale en berbère marocain. La composante rythmique', Cahiers de poétique comparée, 12, 1986, p. 103-127.

MAMMERI M., 'Problèmes de prosodie berbère', Actes du deuxième congrès international d'étude des cultures de la Méditerranée occidentale, T. 2, Alger, SNED, 1078, p. 385-392.

SALHI M.-A., Contribution à l'étude typographique et métrique de la poésie kabyle, Thèse de doctorat, Université de Tizi-Ouzou (Algérie), 2007.

STUMmE H., Dichtung und Gedichte der Shluh, Leipzig, 1895.

INDEX

Mots-clés : Littérature, Poésie 